ESAIM: PROCEEDINGS, April 2009, Vol. 26, p. 100-122

H. Ammari, Editor

\title{
ANISOTROPIC TEXTURE MODELING AND APPLICATIONS TO MEDICAL IMAGE ANALYSIS
}

\author{
Hermine BIERMÉ ${ }^{1}$, Frédéric RICHARD ${ }^{1}$, MOUNA RACHIDI ${ }^{2}$ AND \\ Claude-Laurent Benhamou ${ }^{2}$
}

\begin{abstract}
In this paper, we consider a stochastic anisotropic model for medical image. We model textures by Anisotropic Fractional Brownian Fields (AFBF) which are Gaussian random fields obtained as anisotropic generalizations of the Fractional Brownian Field. The main difficulty with this modeling consists in the estimatation of the anisotropy. We recall here theoretical results obtained in $[2,5]$ to construct consistent estimators for the texture anisotropy analysis. These results allow us to propose statistical tests for deciding if a texture modeled by an AFBF is clearly anisotropic. We apply them to Full-Field Digital Mammograms and show that about 60 percent of textures can be considered as anisotropic with a high level of confidence. We finish this paper with preliminary results on trabecular bone x-ray images. In [4], a fractal analysis based on isotropic Fractional Brownian Fields was proposed to characterize bone microarchitecture. Our first results suggest that anisotropic models are also relevant for the statistical analysis of this microarchitecture.
\end{abstract}

\footnotetext{
1 University Paris Descartes, MAP5, CNRS UMR 8145, 45, rue des Saints-Pères, 75006 Paris, France.

2 INSERM Unit 658, Orleans Hospital, Orleans, France.
}

(C) EDP Sciences, SMAI 2009 
Résumé. Dans cet article, nous nous intéressons à un modèle aléatoire anisotrope pour des images médicales. Nous modélisons les textures par des champs browniens fractionnaires anisotropes (CBFA) qui sont des champs aléatoires gaussiens obtenus comme généralisations anisotropes du champ brownien fractionnaire. L'anisotropie s'estime difficilement avec ce type de modèle. Nous rappelons les resultats théoriques obtenus dans $[2,5]$ pour la construction d'estimateurs consistants afin d'analyser l'anisotropie des textures. Ceci nous a permis d'établir des tests statistiques afin de décider si une texture modélisée par un CBFA est anisotrope. Nous les avons utilisés sur des images de mammographies et avons trouvé qu'environ $60 \%$ d'entre elles peuvent être considérées anisotropes avec un haut niveau de confiance. Nous concluons cet article en donnant quelques résultats préliminaires sur des images radiographiques d'os trabéculaires. Dans [4], une analyse fractale basée sur la modélisation par des champs browniens fractionnaires isotropes a été proposée pour caractériser la microarchitecture osseuse. Nos premiers résultats suggèrent qu'une modélisation par des champs anisotropes est également pertinente pour une analyse statistique de cette microarchitecture.

\section{INTRODUCTION}

In medical image analysis, many authors tried to characterize texture roughness by the fractal dimension of images. For instance, it was used for the characterization and classification of the mammogram density [9] and for the assessment of breast cancer risk. More recently, we [4] linked the fractal dimension of radiographic images with the bone architecture alteration for the evaluation of an osteoporotic fracture risk.

The stochastic model beyond fractal analysis is the Fractional Brownian Field (FBF), which is a multidimensional extension of the famous fractional Brownian motion introduced by Mandelbrot and Van-Ness [20], with fractal dimension determined by its so-called Hurst parameter. However the fractal analysis with FBF models, which is isotropic by definition of $H$, is not completely satisfactory for some applications, since it does 
not reveal texture anisotropy, which can be an important predictor for diagnostic.

Therefore we consider anisotropic generalizations of FBF such as the anisotropic fractional Brownian field [6]. The Hurst parameter is replaced by a function that characterizes the model anisotropy. We briefly recall main properties of these fields in Section 1. Then, in Section 2, we consider anisotropic tests as introduced in [22], based on a parametric estimator constructed in [5]. Finally, in Section 3, we consider two applications to medical images. The first one concerns mammograms. We show that AFBF models are well-suited for the modeling of mammogram texture density: our anisotropic tests reveal that about $60 \%$ of mammograms are not isotropic with a high level of confidence. The second application is related with the problem of early diagnosis for osteoporosis. In [4], fractal analysis of bone radiographs is coupled with bone mineral density to improve the fracture risk evaluation. We present how we applied our estimators to bone radiographs and show some preliminary results emphasizing the anisotropy of the bone microarchitecture.

\section{Anisotropic Fractional Brownian Field}

\subsection{Gaussian fields with stationary increments}

Let $(\Omega, \mathcal{A}, \mathbb{P})$ be a probability space. A $d$-dimensional random field $X$ is a map from $\Omega \times \mathbb{R}^{d}$ into $\mathbb{R}$ such that $X(\cdot, y):=X(y)$ is a real random variable on $\Omega$ for all $y \in \mathbb{R}^{d}$. When $d=1$, such a field is called a random process and can be used to model a signal. We will be mainly interested in the case $d=2$ for which the random field $X$ can stand for the gray level of an image. We say as usual that a random field is Gaussian if any finite linear combination of its associated random variables is a Gaussian variable. A centered Gaussian field $X$ is characterized by its covariance function: $(y, z) \mapsto \operatorname{Cov}(X(y), X(z))$. A field $X$ has stationary increments if the law governing the field $X(\cdot+z)-X(z)$ is the same as $X(\cdot)-X(0)$ for all $z \in \mathbb{R}^{d}$. This property is often used to model homogeneous media. Moreover a centered Gaussian field $X$ with stationary increments is characterized 
by its variogram, which is defined by

$$
\forall y \in \mathbb{R}^{d}, \quad v(y)=\mathbb{E}\left((X(y)-X(0))^{2}\right)
$$

Using a generalization of Bochner's Theorem, some of centered Gaussian fields with stationary increments have a variogram of the form

$$
\forall y \in \mathbb{R}^{d}, v(y)=\int_{\mathbb{R}^{d}}\left|e^{i y \cdot \zeta}-1\right|^{2} f(\zeta) d \zeta
$$

where $f$ is a positive function such that $\int_{\mathbb{R}^{d}}\left(1 \wedge|\zeta|^{2}\right) f(\zeta) d \zeta<\infty$, which is called the spectral density. Such fields are referred to as Gaussian Fields with Spectral Density (GFSD).

In [6], Bonami and Estrade define anisotropic fractional Brownian fields (AFBF) by taking spectral densities which are of the form

$$
\forall \zeta \in \mathbb{R}^{d}, f(\zeta)=|\zeta|^{-2 h(\zeta /|\zeta|)-d}
$$

where $h$ is a map taking values in $(0,1)$ and defined for any direction $\theta$ of the unit sphere $S^{d-1}$ of $\mathbb{R}^{d}$. Due to directional Hurst indices $h$, AFBF have orientation-dependent spectral densities and yield anisotropic fields for non constant $h$.

FBF are particular AFBF, characterized by a density of the form (3) with a constant function $h \equiv H$. The parameter $H \in(0,1)$ is called the Hurst parameter of the FBF $B_{H}$. The variogram of $B_{H}$ given by $(2)$ with $f(\zeta)=|\zeta|^{-2 H-d}$ is simply equal to

$$
v(y)=\operatorname{Var}\left(B_{H}(y)\right)=c_{H, d}|y|^{2 H}
$$

with $c_{H, d}$ a positive constant. It is straightforward to see that the law of $B_{H}$ is then rotation invariant which means that $B_{H}$ is isotropic. Moreover the homogeneity of the variogram induces the famous self-similarity property of the FBF:

$$
\left\{B_{H}(\lambda y) ; y \in \mathbb{R}^{d}\right\} \stackrel{f d d}{=} \lambda^{H}\left\{B_{H}(y) ; y \in \mathbb{R}^{d}\right\},
$$


where $f d d$ means equality for finite dimensional distributions. Lots of fractal properties follow from this scaling behavior. In particular, using Kolmogorov-Centsov criterion [15], one can prove that $H$ is the critical Hölder exponent of $B_{H}$. This means that for any $\alpha \in(0, H)$ the sample paths of $B_{H}$ satisfy a uniform Hölder condition of order $\alpha$ on $[-T, T]^{d}$, for any $T>0$ : there exists a positive random variable $A$ with $\mathbb{P}(A<+\infty)=1$ such that

$$
\forall y, z \in[-T, T]^{d}, \quad\left|B_{H}(y)-B_{H}(z)\right| \leq A|y-z|^{\alpha} ;
$$

while for any $\alpha \in(H, 1)$, almost surely the sample paths of $B_{H}$ fail to satisfy any uniform Hölder condition of order $\alpha$.

From an image point of view $(d=2)$, the critical Hölder exponent is related to the roughness of the texture. The texture is as rough as the field regularity $H$ is small.

Note also that the critical Hölder exponent $H$ of a FBF $B_{H}$ is related to the Hausdorff and Box-counting fractal dimensions of its graph $\mathcal{G}\left(B_{H}\right)=\left\{\left(y, B_{H}(y)\right) ; y \in[-T, T]^{d}\right\}$ by

$$
\operatorname{dim}_{\mathcal{H}} \mathcal{G}\left(B_{H}\right)=\operatorname{dim}_{\mathcal{B}} \mathcal{G}\left(B_{H}\right)=d+1-H
$$

almost surely, for any $T>0$ (see [11] for the dimension definitions and proofs).

Actually, in the more general setting of GFSD, all this properties can be deduced from the local behavior of the variogram around $0(7)$ or from the asymptotic behavior of the spectral density at high-frequencies (6).

\subsection{Hölder regularity}

Let us introduce some useful notations. For any $H \in(0,1)$, if $f$ is a spectral density satisfying: for any $\varepsilon>0$ there exists $A, B_{1}, B_{2}>0$ and a positive-measure subset $E$ of $S^{d-1}$ such that for almost all $\xi \in \mathbb{R}^{d}$,

$$
|\xi| \geq A \Rightarrow B_{2}|\xi|^{-2 H-d-\varepsilon} \mathbf{1}_{E}\left(\frac{\xi}{|\xi|}\right) \leq f(\xi) \leq B_{1}|\xi|^{-2 H-d+\varepsilon}
$$


we note $f(\xi) \underset{+\infty}{\asymp}|\xi|^{-2 H-d}$. In the same vein, for any $H \in(0,1)$, if $v$ is a variogram satisfying: for any $\varepsilon>0$ there exists $\delta, C_{1}, C_{2}>0$ such that for all $y \in \mathbb{R}^{d}$,

$$
|y| \leq \delta \Rightarrow C_{2}|y|^{2 H+\varepsilon} \leq v(y) \leq C_{1}|\xi|^{2 H-\varepsilon},
$$

we note $v(y) \underset{0}{\asymp}|y|^{2 H}$. We recall here Theorem 2.1 of [22], proved in [6].

Theorem 1.1. Let $X$ be a GFSD with spectral density $f$ and variogram $v$. Let $H \in(0,1)$.

(a) If $f(\xi) \underset{+\infty}{\asymp}|\xi|^{-2 H-d}$ then $v(y) \underset{0}{\asymp}|y|^{2 H}$.

(b) If $v(y) \underset{0}{\asymp}|y|^{2 H}$ then $X$ admits $H$ as critical Hölder exponent.

In particular, this shows that the critical Hölder exponent of $X$ an AFBF is equal to the minimal value $H$ of the Hurst index function $h$ on $S^{d-1}$ :

$$
H=\underset{S^{d-1}}{\operatorname{essinf}}(h)
$$

since it is straightforward to see that its spectral density given by (3) satisfies (a) of Theorem 1.1. The critical Hölder exponent $H$ of an AFBF will be called the minimal Hurst index. Let us mention that an AFBF will also satisfy (5) with $H$ its minimal Hurst index. Therefore the minimal Hurst index characterizes the AFBF texture roughness, but being direction-independent, it does not capture any anisotropic feature of an AFBF.

In attempt to characterize the anisotropy of an AFBF, one could propose to study the field along oriented straight lines. Let $X$ be a a GFSD defined on $\mathbb{R}^{d}$, then for any direction $\theta \in S^{d-1}$ and $y_{0} \in \mathbb{R}^{d}$ the restriction $\left\{X\left(y_{0}+t \theta\right) ; t \in \mathbb{R}\right\}$ of $X$ along the line $y_{0}+\mathbb{R} \theta$ defines a Gaussian process with spectral density (GFSD with $d=1$ ) given by

$$
\forall p \in \mathbb{R}, f_{\theta}(p)=\int_{\langle\theta\rangle \perp} f(\xi+p \theta) d \xi,
$$

where $f$ is the spectral density of $X$ and $\langle\theta\rangle^{\perp}$ denotes the hyperplane of $\mathbb{R}^{d}$ orthogonal to the direction $\theta$. Let us remark that $(\theta, p) \mapsto f_{\theta}(p)$ is the Radon transform of $f$ (see [21] p.11 for instance). Moreover, if $X$ is an AFBF with directional Hurst index $h$ one has $f_{\theta}(p) \underset{+\infty}{\asymp}|p|^{-2 H-1}$ for $H$ its minimal Hurst index given by (8). 


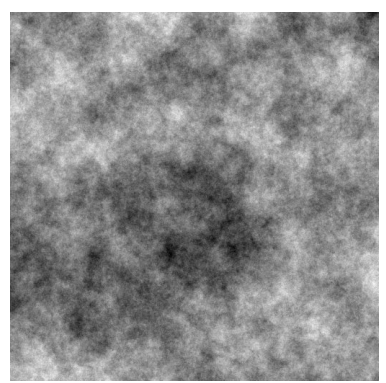

(a)

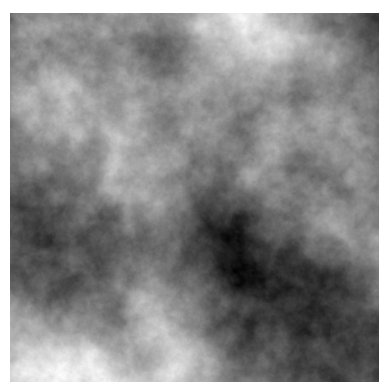

(b)

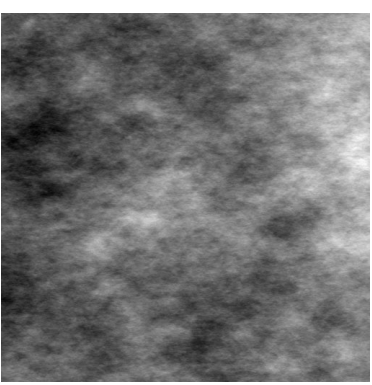

(c)

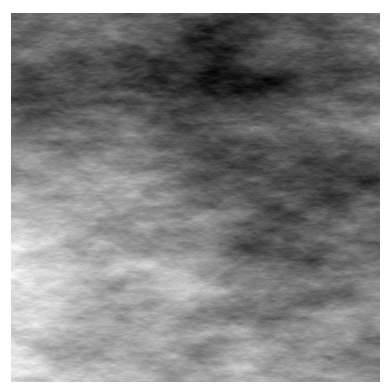

(d)

FigurE 1. Simulation of FBF by the Stein method for (a) $h=0.3$ and (b) $h=0.7$. Simulation of AFBF by the spectral method for (c) $h_{1}=0.3$ and $h_{2}=0.5$ and (d) $h_{1}=0.3$ and $h_{2}=0.7$.

Therefore Theorem 1.1 implies that the critical Hölder exponent $H_{\theta}$ of the process $\left\{X\left(t_{0}+t \theta\right) ; t \in \mathbb{R}\right\}$ equals $H$ in any direction $\theta$.

In order to reveal anisotropy, the authors of [6] proposed to study windowed Radon transforms. For $X$ a GFSD defined on $\mathbb{R}^{d}$ and any direction $\theta \in S^{d-1}$, they give a rigorous definition of the process $R_{\theta} X$ obtained as windowed Radon transform of $X$ in the direction $\theta$

$$
\forall t \in \mathbb{R}, \quad R_{\theta} X(t)=\int_{\langle\theta\rangle \perp} X(s+t \theta) \rho(s) d s,
$$

where $\rho$ is a window function of the Schwartz class. $R_{\theta} X$ also defines a Gaussian process with spectral density given by

$$
\forall p \in \mathbb{R}, \mathcal{R}_{\theta} f(p)=\int_{\langle\theta\rangle \perp} f(\xi+p \theta)|\widehat{\rho}(\xi)|^{2} d \xi
$$

Note that this transform has a smoothing effect and we use an extended definition of the Hölder regularity which has a meaning when $H \geq 1[6]$. We say that a Gaussian process $Y=\{Y(t) ; t \in \mathbb{R}\}$ admits $H \in(n, n+1)$ as critical Hölder exponent for some $n \in \mathbb{N}$ if $Y$ is $n$ times mean square differentiable in the sense of [1] and the process $Y^{(n)}$ admits $H$ as critical Hölder exponent. Then Theorem 2.2 of [22] can be formulated as follow. 
Theorem 1.2. Let $Y$ be a Gaussian process with spectral density $f$ and variogram $v$. Let $H \in(n, n+1), n \in \mathbb{N}$.

(a) If $f(p) \underset{+\infty}{\asymp}|p|^{-2 H-1}$ then $v \in \mathcal{C}^{2 n}(\mathbb{R})$ and $\left|v^{(2 n)}(y)-v^{(2 n)}(0)\right| \underset{0}{\asymp}|y|^{2(H-n)}$.

(b) If $v \in \mathcal{C}^{2 n}(\mathbb{R})$ and $\left|v^{(2 n)}(y)-v^{(2 n)}(0)\right| \underset{0}{\asymp}|y|^{2(H-n)}$ then $Y$ admits $H$ as critical Hölder exponent.

Proposition 2.1 of [5] shows that when $X$ is an AFBF with directional Hurst index $h$ one has $\mathcal{R}_{\theta} f(p) \underset{+\infty}{\longleftarrow}$ $|p|^{-2 h(\theta)-d}$ such that $R_{\theta} X$ admits $h(\theta)+\frac{d-1}{2}$ as critical Hölder exponent.

\section{Estimation AND ANISOTROPY TEST}

Among numerous estimators constructed to estimate the Hurst parameter of a fractional Brownian motion we choose estimators based on generalized quadratic variations as studied in $[14,16]$.

\subsection{Generalized quadratic variations}

Let $H \in(0,1)$ and $B_{H}$ a fractional Brownian motion on $\mathbb{R}$. We assume to observe $N+1$ equispaced points of its sample path, namely $\left\{B_{H}\left(\frac{p}{N}\right) ; 0 \leq p \leq N-1\right\}$. The fractional Brownian motion is not stationary but any of its increments is. The main idea of generalized quadratic variations is to use adaptations of ergodic Theorem which states that if $\left(X_{p}\right)_{p \in \mathbb{N}}$ is an ergodic stationary sequence of mean square integrable random variables then for $S_{n}=\sum_{p=0}^{n-1} X_{p}$ we have

$$
\frac{S_{n}}{n} \underset{n \rightarrow+\infty}{\longrightarrow} \mathbb{E}\left(X_{0}\right) \text { a.s. with } \sqrt{n}\left(\frac{S_{n}}{n}-\mathbb{E}\left(X_{0}\right)\right) \underset{n \rightarrow+\infty}{\stackrel{d}{\longrightarrow}} \mathcal{N}(0,1) \text {. }
$$

For any $a \in \mathbb{N}$ with $a \geq 1$, considering $X_{p}^{a}:=\left(B_{H}\left(\frac{p}{N}+\frac{a}{N}\right)-B_{H}\left(\frac{p}{N}\right)\right)^{2}$ for any $p \in \mathbb{N}$, the sequence $\left(X_{p}^{a}\right)_{p \in \mathbb{N}}$ is stationary and $\mathbb{E}\left(X_{0}^{a}\right)=\mathbb{E}\left(B_{H}\left(\frac{a}{N}\right)^{2}\right)=v\left(\frac{a}{N}\right)=c_{H, 1} a^{2 H} N^{-2 H}$. However this sequence is ergodic only if $H<3 / 4$. One can overcome this restriction by considering second-order increments (see [14] for instance).

For $Y=\{Y(t) ; t \in \mathbb{R}\}$ a Gaussian process with spectral density we consider the stationary sequence of second-order increments of $Y$ with step $a$ as

$$
Z_{N, a}(Y)(p)=Y\left(\frac{p}{N}+2 \frac{a}{N}\right)-2 Y\left(\frac{p}{N}+\frac{a}{N}\right)+Y\left(\frac{p}{N}\right)
$$


The stationary sequence $\left(\left(Z_{N, a}\left(B_{H}\right)(p)\right)^{2}\right)_{p \in \mathbb{N}}$ is ergodic for any $H \in(0,1)$ and this can be generalized to $Y$ instead of $B_{H}$, provided that the spectral density $f$ of $Y$ satisfies $f(p) \underset{+\infty}{\asymp}|p|^{-2 H-1}$ for some $H<7 / 4$. Therefore we will consider the generalized quadratic variations of $Y$ defined as

$$
V_{N, a}(Y)=\frac{1}{N-2 a} \sum_{p=0}^{N-2 a-1}\left(Z_{N, a}(Y)(p)\right)^{2},
$$

from the observation of $\left\{Y\left(\frac{p}{N}\right) ; 0 \leq p \leq N-1\right\}$. A natural estimator of $H$ is then given by

$$
\widehat{H}_{N}=\frac{1}{2 \log (2)} \log \left(\frac{V_{N, 2}(Y)}{V_{N, 1}(Y)}\right)
$$

We refer to [14], respectively Proposition 1.3 of [5], for assumptions on the variogram, or on the spectral density of $Y$ to ensure the convergence of (14) to $H$ with asymptotic normality.

\subsection{Anisotropy test}

In this section we consider $X=\left\{X(y) ; y \in \mathbb{R}^{2}\right\}$ an AFBM in $\mathbb{R}^{2}$ with a spectral density $f$ satisfying (3) for some function $h$ such that for any $\theta=\left(\theta_{1}, \theta_{2}\right) \in S^{1} \subset \mathbb{R}^{2}$,

$$
h(\theta)= \begin{cases}h_{1}, & \text { if }\left|\theta_{1}\right|<c_{1}\left|\theta_{2}\right| \\ h_{2} & \text { if }\left|\theta_{2}\right|<c_{2}\left|\theta_{1}\right|\end{cases}
$$

with $0<c_{2} \leq c_{1}^{-1}$. We assume to observe $\left\{X\left(\frac{p_{1}}{N}, \frac{p_{2}}{N}\right) ; 0 \leq p_{1}, p_{2} \leq N-1\right\}$ for some $N \geq 1$. For $0 \leq p \leq N-1$, we write $X_{1}^{p}$ for the process $\left\{X\left(\frac{p}{N}, t\right) ; t \in \mathbb{R}\right\}$ obtained by restriction of $X$ along the vertical line going through $\left(\frac{p}{N}, 0\right)$. Similarly, we write $X_{2}^{p}$ for the process $\left\{X\left(t, \frac{p}{N}\right) ; t \in \mathbb{R}\right\}$ obtained by restriction of $X$ along the horizontal line going through $\left(0, \frac{p}{N}\right)$. From Proposition 1.3 of [5], for any $e \in\{1,2\}$ and $0 \leq p \leq N-1$,

$$
\frac{1}{2 \log (2)} \log \left(\frac{V_{N, 2}\left(X_{e}^{p}\right)}{V_{N, 1}\left(X_{e}^{p}\right)}\right) \underset{N \rightarrow+\infty}{\stackrel{a . s .}{\rightarrow}} H, \text { with } \sqrt{N}\left(\frac{1}{2 \log (2)} \log \left(\frac{V_{N, 2}\left(X_{e}^{p}\right)}{V_{N, 1}\left(X_{e}^{p}\right)}\right)-H\right) \underset{N \rightarrow+\infty}{\stackrel{d}{\longrightarrow}} \mathcal{N}\left(0, \sigma_{H}^{2}\right),
$$

where $H=\underset{S^{1}}{\operatorname{essinf}}(h) \leq \min \left(h_{1}, h_{2}\right)$ according to $(8), \sigma_{H}^{2}$ is a positive constant that only depends on $H, \stackrel{a . s .}{\longrightarrow}$ means the almost sure convergence and $\stackrel{d}{\longrightarrow}$ means the convergence in distribution. Actually we can consider

$$
\hat{H}_{0 e}=\frac{1}{2 \log (2)} \log \left(\sum_{p=0}^{N-1} V_{N, 2}\left(X_{e}^{p}\right) / \sum_{p=0}^{N-1} V_{N, 1}\left(X_{e}^{p}\right)\right)
$$


and establish the following result.

Proposition 2.1. Let $X$ be an $A F B F$ with directional Hurst index $h$ satisfying (15). Let $N \geq 1$ and $e \in\{1,2\}$. Then, for $H=\underset{S^{1}}{\operatorname{essinf}}(h)$ and some $\gamma>0$, we get

$$
\hat{H}_{0 e} \underset{N \rightarrow+\infty}{\stackrel{a . s .}{\longrightarrow}} H \text {, with } N\left(\hat{H}_{0 e}-H\right) \underset{N \rightarrow+\infty}{\stackrel{d}{\longrightarrow}} \mathcal{N}\left(0, \gamma_{H}^{2}\right)
$$

We use windowed Radon transform (10) to estimate $h_{1}$ and $h_{2}$. For $\rho \in \mathcal{S}(\mathbb{R})$ a window, let us denote $R_{1} X$, respectively $R_{2} X$, the windowed Radon transform of $X$ in the direction $(0,1)$, respectively in the direction $(1,0)$, as defined by (10). From Theorem 2.3 of [5] and Theorem 3.1 of [22] we can consider the following estimators defined for $e \in\{1,2\}$.

$$
\widehat{h}_{e}=\frac{1}{2 \log (2)} \log \left(\frac{V_{N, 2}\left(R_{e} X\right)}{V_{N, 1}\left(R_{e} X\right)}\right)-\frac{1}{2} .
$$

Proposition 2.2. Let $X$ be an $A F B F$ with directional Hurst index $h$ satisfying (15). Let $N \geq 1$ and $e \in\{1,2\}$. Then, for some $\sigma_{h_{e}}>0$,

$$
\widehat{h}_{e} \underset{N \rightarrow+\infty}{\stackrel{a . s .}{\longrightarrow}} h_{e}, \text { with } \sqrt{N}\left(\widehat{h}_{e}-h_{e}\right) \underset{N \rightarrow+\infty}{\stackrel{d}{\longrightarrow}} \mathcal{N}\left(0, \sigma_{h_{e}}^{2}\right) \text {. }
$$

Moreover, for some $\gamma>0$

$$
\widehat{h}_{1}-\widehat{h}_{2} \underset{N \rightarrow+\infty}{\stackrel{\text { a.s. }}{\longrightarrow}} h_{1}-h_{2} \text {, with } \sqrt{N}\left(\widehat{h}_{1}-\widehat{h}_{2}-\left(h_{1}-h_{2}\right)\right) \underset{N \rightarrow+\infty}{\stackrel{d}{\longrightarrow}} \mathcal{N}\left(0, \gamma^{2}\right) \text {. }
$$

We can therefore consider the statistical asymptotic hypothesis test for testing the assumption $\mathbf{H}_{0}: h_{1}=h_{2}$ (isotropy) against $\mathbf{H}_{1}: h_{1} \neq h_{2}$ (anisotropy). Our test statistic is naturally defined by

$$
D_{N}=\sqrt{N}\left|\hat{h}_{1}-\hat{h}_{2}\right|
$$

By Proposition 2.1, under the assumption $\mathbf{H}_{0}$ we have $D_{N} \underset{N \rightarrow+\infty}{\stackrel{d}{\longrightarrow}}\left|\mathcal{N}\left(0, \gamma^{2}\right)\right|$ while $D_{N} \underset{N \rightarrow+\infty}{\longrightarrow}+\infty$ under the assumption $\mathbf{H}_{1}$. Hence, we define the form of an hypothesis rejection interval (of confidence level $\alpha$ ) as

$$
\mathcal{R}_{N}(\alpha)=\left\{D_{N}>\gamma c_{\alpha}\right\}
$$

where $c_{\alpha}$ stands for $(1-\alpha / 2)$-quantile of the centered and normalized Gaussian distribution. 


\subsection{Implementation}

We consider an image $(I(n, m))_{0 \leq p_{1}, p_{2} \leq N-1}$ of size $N \times N$ as a realization of an AFBM over a grid $\left\{X\left(\frac{p_{1}}{N}, \frac{p_{2}}{N}\right) ; 1 \leq p_{1}, p_{2} \leq N\right\}$ with spectral density satisfying (15).

As in [2], we approximate $R_{1} X$, resp. $R_{2} X$, the windowed Radon transform of $X$ in the direction $(0,1)$, resp. $(1,0)$., by $Y_{1}$, resp. $Y_{2}$, the discrete row, resp. column, average of the observations of $X$ :

$$
Y_{1}\left(\frac{p_{2}}{N}\right)=\sum_{p_{1}=0}^{N-1} X\left(\frac{p_{1}}{N}, \frac{p_{2}}{N}\right), \text { resp. } Y_{2}\left(\frac{p_{1}}{N}\right)=\sum_{p_{2}=0}^{N-1} X\left(\frac{p_{1}}{N}, \frac{p_{2}}{N}\right)
$$

for any $0 \leq p_{1}, p_{2} \leq N-1$. Due to the Hölder regularity of $X$, we can find an upper bound for the error of the Radon's transform discretization. Actually, for any $r<H=\underset{S^{1}}{\operatorname{essinf}}(h)$, one can find $C>0$ such that almost surely, for any $e \in\{1,2\}$ and $0 \leq p \leq N-1$,

$$
\left|R_{e} X\left(\frac{p}{N}\right)-Y_{e}\left(\frac{p}{N}\right)\right| \leq C N^{-r}
$$

In order to neglect this error compared to the estimation of $h_{e}+\frac{1}{2}$, which is the regularity of $R_{e} X$, the trick is to subsample $Y_{e}$. We note $V_{\nu, a}(\cdot)$ instead of $V_{N / 2^{\nu}, a}(\cdot)$, for $\nu \leq \log _{2}(N)$. Following the same lines as [2], we can prove that for any $r<H=\underset{S^{1}}{\operatorname{essinf}}(h)$, one can find $C>0$ such that almost surely, for any $e, a \in\{1,2\}$

$$
\left|\log \left(\frac{V_{\nu, a}\left(Y_{e}\right)}{V_{\nu, a}\left(R_{e} X\right)}\right)\right| \leq C N^{-r}\left(N / 2^{\nu}\right)^{h_{e}+1 / 2}
$$

which corresponds to the error of approximation, considering

$$
\hat{h}_{e}^{\nu}=\frac{1}{2 \log (2)} \log \left(\frac{V_{\nu, 2}\left(Y_{e}\right)}{V_{\nu, 1}\left(Y_{e}\right)}\right)-\frac{1}{2}
$$

instead of $\widehat{h}_{e}$ given by (17). We can define an empirical statistical test for testing the assumption $\mathbf{H}_{0}: h_{1}=h_{2}$ (isotropy) against $\mathbf{H}_{1}: h_{1} \neq h_{2}$ (anisotropy). Let $\hat{d}^{\nu}=\left|\hat{h}_{1}^{\nu}-\hat{h}_{2}^{\nu}\right|$ be the empirical approximation of the statistic $\frac{D_{N / 2^{\nu}}}{\sqrt{N / 2^{\nu}}}$ in Equation (18), with $\hat{h}_{1}^{\nu}$ and $\hat{h}_{2}^{\nu}$ given by (21). The rejection interval of confidence level $\alpha=0.05$ for the above test is defined as

$$
\mathcal{R}^{\nu}=\left\{\hat{d}^{\nu}>1.96 \sigma^{\nu}\right\}
$$


where 1.96 is the 0.975 -quantile of the centered and normalized Gaussian distribution and $\sigma^{\nu}=\frac{\gamma}{\sqrt{N / 2^{\nu}}}$ is the standard deviation of $\hat{d}^{\nu}$. However, Proposition 2.1 does not give an explicit value of $\gamma$, nor the optimal choice for $\nu$. We set empirically these two parameters [5,22], considering exact synthesis for fractional Brownian fields obtained using Stein method [23]. We evaluated the accuracy and the precision of each estimator on a dataset of 7 subsets of $1000 \mathrm{FBF}$ of size $512 \times 512$ generated using Stein method for $H \in\{0.1, \ldots, 0.7\}$. The accuracy of an estimator on a given set was obtained by averaging parameter estimates over the set and subtracting the true parameter value; it is the empirical bias of the estimator. The precision of an estimator on a given set was defined as the standard deviation of parameter estimates over the set. Accuracy and precision are both reported in Figures 2.
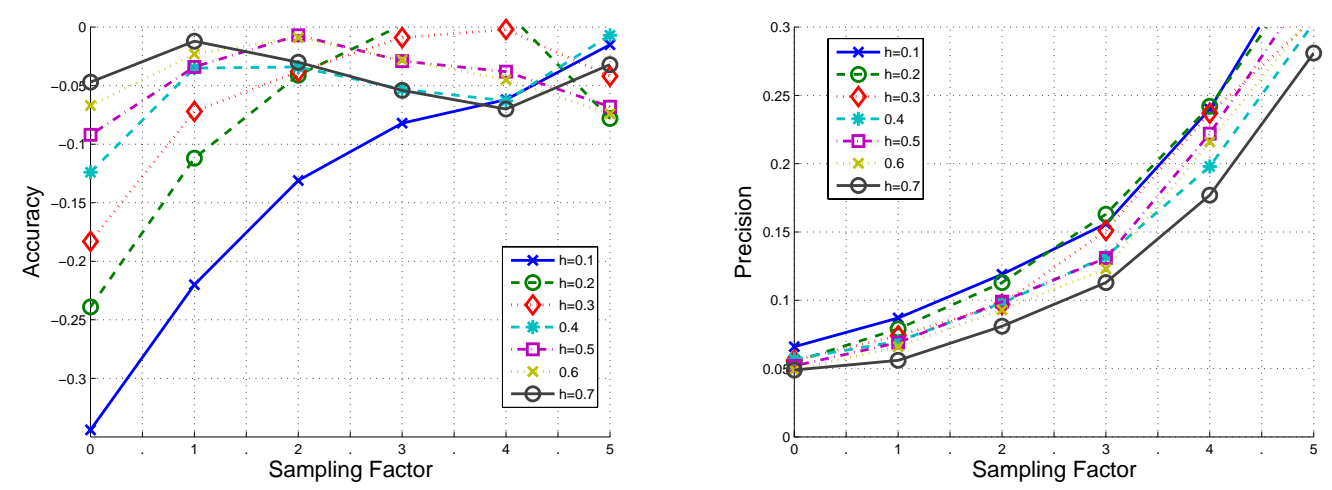

Figure 2. Projection-based estimation of global Hurst index on FBF simulated using the Stein method. The accuracy and precision of the projection-based estimators $\hat{h}_{e}^{\nu}(e=1,2)$ are computed for different global Hurst index values of fields and different estimator sampling factors.

Figure 2 shows that accuracy and precision both depends on the estimator sampling factor. The precision becomes worse and worse as the sampling factor increases. However, when the sub-sampling factor is fixed, the precision does not vary significantly when values of the estimated index are changed. For any fixed sampling factor below 4 , the precision variations are of order 0.05 . Concerning the accuracy, estimators tend to underestimate the real index value and the underestimation bias increases as the index value decreases, which is due 
to the discretization of the Radon transform. For any estimated index value $H$, the accuracy further varies as a function of the sampling factor and reaches an optimum for a sampling factor value $\nu_{\text {opt }}(h)$. The accuracy optima are almost the same for the different index values. However, the optimal sampling values $\nu_{\mathrm{opt}}(h)$ are different: it is higher for low index values than for high index values. Considering index values altogether, the best accuracy seems to be reached for $\nu=2$. In this case, the estimation error is below 0.05 for all index values varying between 0.2 and 0.8 and around 0.13 for the index value 0.1 . The precision achieved for the same sampling factor $\nu=2$ is about 0.1 . The sampling factor $\nu=2$ gives a good compromise between accuracy and precision and will be used to measure directional indices in applications.

For comparison, Table 1 shows the accuracy and the precision of the line-based estimator for the Hurst index given by (16). We evaluated the accuracy and precision of the estimation of the Hurst index difference by the

\begin{tabular}{|c|c|c|c|c|}
\hline & \multicolumn{2}{|c|}{$\widehat{H}_{01}$} & \multicolumn{2}{c|}{$\widehat{H}_{02}$} \\
\hline Hurst & Accuracy & Precision & Accuracy & Precision \\
\hline$H=0.7$ & $<10 e^{-3}$ & 0.0017 & -0.001 & 0.014 \\
$H=0.5$ & $<10 e^{-3}$ & 0.0016 & $<10 e^{-3}$ & 0.017 \\
$H=0.2$ & 0.003 & 0.0019 & 0.001 & 0.0018 \\
\hline
\end{tabular}

TABLE 1. Line-based estimation of global Hurst index on FBF simulated using the Stein method.

estimators $\hat{d}^{\nu}=\left|\hat{h}_{1}^{\nu}-\hat{h}_{2}^{\nu}\right|$, for $\nu=0,2$, see Figure 3. At the opposite of results of Figure 2 for a single estimator, accuracy and precision do not vary significantly with $H$. Since precision is better for $\nu=0$ (around 0.8 ) we consider the rejection interval given by

$$
\mathcal{R}^{0}=\left\{\hat{d}^{0}>0.16\right\}
$$

We reported in Table 2 the value $p$ of percentage of simulations for which we can reject isotropy assumption $\mathbf{H}_{0}$, evaluated on exact synthesis of $\mathrm{FBF}\left(h_{1}=h_{2}\right)$ and on approximated simulations of $\operatorname{AFBM}\left(h_{1} \neq h_{2}\right)$. On isotropic simulations, tests produce few errors, whatever the value of the minimal Hurst index, but results are slightly better when the Hurst index is high. On anisotropic simulations, the test is not efficient when Hurst index differences are below 0.2 (between 32 and $43 \%$ of errors). However, it becomes reliable when differences 


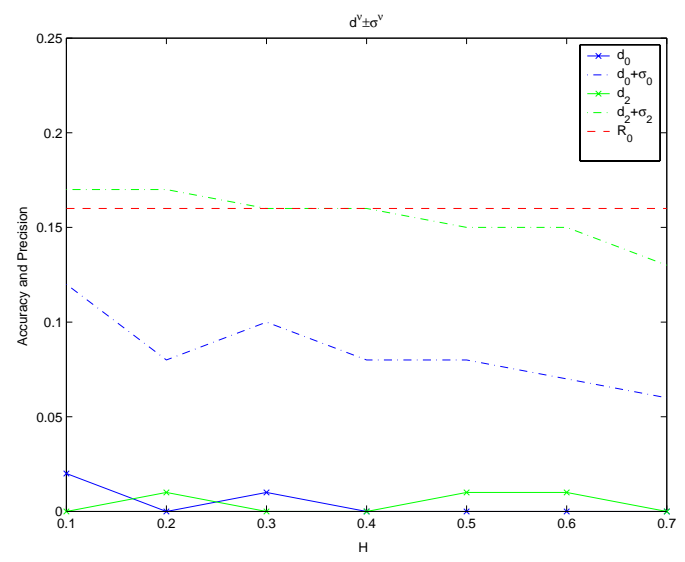

FiguRE 3. Hurst index difference estimation $\hat{d}^{\nu}=\left|\hat{h}_{1}^{\nu}-\hat{h}_{2}^{\nu}\right|$ on FBF simulated using the Stein method.

\begin{tabular}{|c|c|c|c|c|c|c|c|c|c|c|c|c|c|}
\hline$\left|h_{1}-h_{2}\right|$ & \multicolumn{9}{|c|}{0} & \multicolumn{3}{|c|}{0.2} & \multicolumn{3}{|c|}{0.4} & 0.6 \\
\hline$h_{1}$ & 0.1 & 0.2 & 0.3 & 0.4 & 0.5 & 0.6 & 0.7 & 0.5 & 0.7 & 0.9 & 0.7 & 0.9 & 0.9 \\
\hline$h_{2}$ & 0.1 & 0.2 & 0.3 & 0.4 & 0.5 & 0.6 & 0.7 & 0.3 & 0.5 & 0.7 & 0.3 & 0.5 & 0.3 \\
\hline$p$ & 11 & 5 & 7 & 4 & 3 & 2 & 1 & 59 & 57 & 68 & 100 & 100 & 100 \\
\hline
\end{tabular}

TABLE 2. Percentage of simulations for which we can reject isotropy assumption $\mathbf{H}_{0}$ according to $(23)$, evaluated on exact synthesis of FBF $\left(h_{1}=h_{2}\right)$ and on approximated simulations of $\operatorname{AFBM}\left(h_{1} \neq h_{2}\right)$.

are above 0.4 ( $0 \%$ of errors).

This test evaluates the anisotropy between vertical and horizontal directions. Hence it cannot detect anisotropic fields which have the same vertical and horizontal directional Hurst indices. In order to fix this drawback, we set a second anisotropy test which takes into account the other directions using an estimation of the minimal directional Hurst index $H$ : we test the assumption $\mathbf{H}_{0}: H=h_{1}=h_{2}$ (isotropy) against $\mathbf{H}_{1}$ : $H \neq h_{1}$ or $H \neq h_{2}$ (anisotropy). We consider the empirical statistic $\hat{\delta}^{\nu}=\left|\max \left(\hat{h}_{1}^{\nu}, \hat{h}_{2}^{\nu}\right)-\hat{H}\right|$, with $\hat{h}_{1}^{\nu}, \hat{h}_{2}^{\nu}$ given 
by $(21)$ and $\hat{H}=\min \left(\widehat{H_{01}}, \widehat{H_{02}}\right)$ given by (16). The rejection interval of the test is then defined by

$$
R^{\nu}=\left\{\hat{\delta}^{\nu}>c\right\},
$$

where $c$ is a positive constant which will be fixed empirically, under assumption $\mathbf{H}_{0}$.

We evaluated the accuracy and precision of the estimators $\hat{\delta}^{\nu}=\left|\max \left(\hat{h}_{1}^{\nu}, \hat{h}_{2}^{\nu}\right)-\hat{H}\right|$, for $\nu=0,2$, see Figure

4. The mean value of the statistic $\hat{\delta}^{\nu}$ obtained for $\nu=0$ increases as the value of $h$ decreases, while, for $\nu=2$,

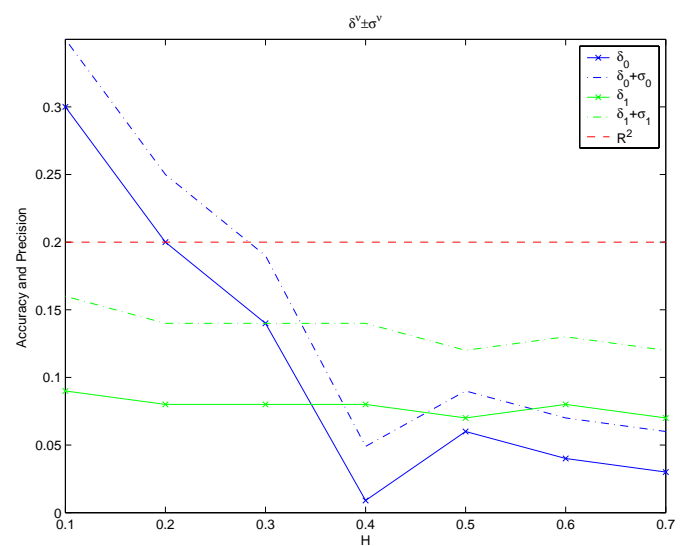

Figure 4. Hurst index difference estimation $\hat{\delta}^{\nu}=\left|\max \left(\hat{h}_{1}^{\nu}, \hat{h}_{2}^{\nu}\right)-\hat{H}\right|$ on FBF simulated using the Stein method.

it remains constant around 0.8. This is due to the bias of the estimators $\hat{h}_{1}^{\nu}$ and $\hat{h}_{2}^{\nu}$. The standard deviation does not vary significantly according to $h$. However, we observe that they are slightly lower than the standard deviations of the previous statistics $\hat{d}^{\nu}$. Since accuracy is better for $\nu=2$ we consider the rejection interval given by

$$
R^{2}=\left\{\hat{\delta}^{2}>0.2\right\} .
$$

We reported in Table 3 the value $p$ of percentage of simulations for which we can reject isotropy assumption $\mathbf{H}_{0}$, evaluated on exact synthesis of $\mathrm{FBF}\left(h_{1}=h_{2}=H\right)$ and on approximated simulations of AFBM $\left(\max \left(h_{1}, h_{2}\right) \neq\right.$ $\left.\min \left(h_{1}, h_{2}\right)=H\right) . \quad$ On isotropic simulations, tests produce few errors, whatever the value of the minimal Hurst index, but as for the first test, results are slightly better when the Hurst index is high. On anisotropic 


\begin{tabular}{|c|c|c|c|c|c|c|c|c|c|c|c|c|c|}
\hline $\max \left(h_{1}, h_{2}\right)-H$ & \multicolumn{9}{|c|}{0} & \multicolumn{3}{|c|}{0.2} & \multicolumn{2}{|c|}{0.4} & 0.6 \\
\hline$h_{1}$ & 0.1 & 0.2 & 0.3 & 0.4 & 0.5 & 0.6 & 0.7 & 0.5 & 0.7 & 0.9 & 0.7 & 0.9 & 0.9 \\
\hline$h_{2}$ & 0.1 & 0.2 & 0.3 & 0.4 & 0.5 & 0.6 & 0.7 & 0.3 & 0.5 & 0.7 & 0.3 & 0.5 & 0.3 \\
\hline$p$ & 9 & 7 & 4 & 7 & 3 & 3 & 1 & 7 & 14 & 22 & 64 & 83 & 83 \\
\hline
\end{tabular}

TABLE 3. Percentage of simulations for which we can reject isotropy assumption $\mathbf{H}_{0}$ according to $(24)$, evaluated on exact synthesis of $\operatorname{FBF}\left(h_{1}=h_{2}=H\right)$ and on approximated simulations of $\operatorname{AFBM}\left(\max \left(h_{1}, h_{2}\right) \neq \min \left(h_{1}, h_{2}\right)=H\right)$.

simulations, the test is not efficient when Hurst index differences are below 0.2. However, it becomes reliable when differences are above 0.4 .

\section{Application to medical images}

\subsection{Mammogram}

In this section, we apply this methodology on mammograms and study the relevance of an anisotropic model for the characterization of the textures of these images. Our database has a total of 58 cases, each case being composed of Full-Field Digital Mammograms (FFDM) of the left and right breasts of a woman. Images were acquired in MLO position using a Senographe 2000D (General Electric Medical Systems, Milwaukee, WI), with a spatial resolution of $0.1 \mathrm{~mm}^{2}$ per pixel (image size: $1914 \mathrm{x} 2294$ pixels). Images are courtesy of the Department of Radiology of the University of Pennsylvania. In each image of the database, we extracted manually a region of interest $(\mathrm{ROI})$ of size $512 \times 512$ within the densest region of the breast. As illustrated on Figure 5 , we then computed the discrete row- and column-wise projections of each ROI and the estimates of the directional Hurst indices in both directions $\left(\hat{h}_{1}^{\nu}, \hat{h}_{2}^{\nu}\right.$ for $\nu=0,2$ given by $\left.(21)\right)$. We also estimated the minimal Hurst index $\hat{H}=\min \left(\hat{H_{01}}, \hat{H_{02}}\right)$ using the line-based estimators given in (16). Note that in mammograms, vertical (row) and horizontal (column) directions (labeled 1 and 2) correspond to directions perpendicular and parallel to the chest wall, respectively. 

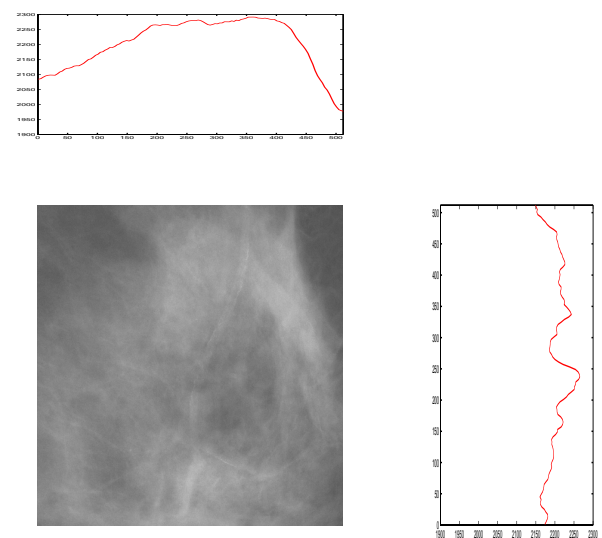

FIgURE 5. A ROI extracted from a FFDM and its vertical and horizontal projections $\left(\hat{h}_{1}^{2}=\right.$ $0.73, \hat{h}_{2}^{2}=0.47$ and $\left.\hat{H}=0.26\right)$.

The estimates of the minimal Hurst index we obtained using line-based estimators on the extracted ROI are in the interval $[0.18 ; 0.42]$, with an average of 0.31 and a standard deviation of 0.05 . These results are
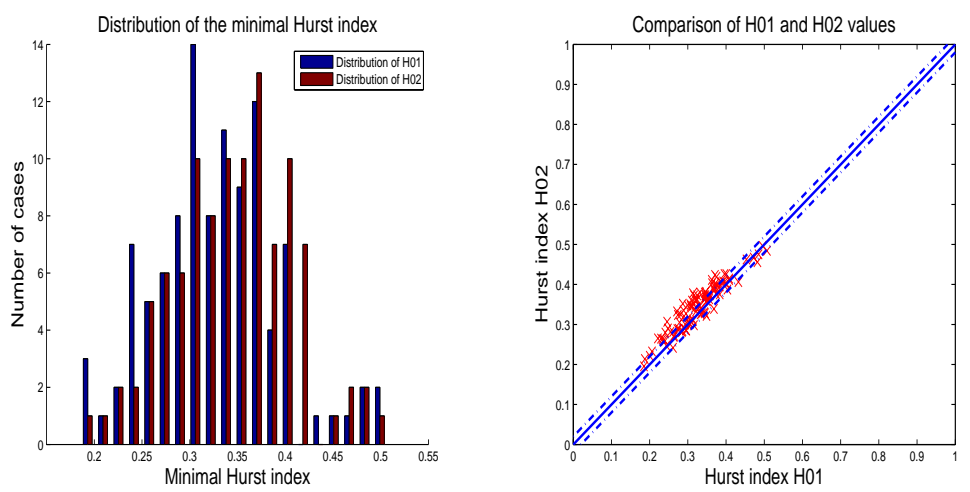

Figure $6 . \hat{H}=\min \left(\hat{H}_{01}, \hat{H}_{02}\right)=0.31 \pm 0.05$

close to those obtained in previous studies, using other estimators. For instance, Kestener et al. computed the Hurst index on small regions of size $512 \times 512$ of film mammograms taken from the DDSM database [17]. The values of the minimal Hurst index are in $[0.20 ; 0.35]$ and $[0.55 ; 0.75]$ for ROI with predominant adipose tissues, respectively. In [13], authors used a spectral method for the estimation of the regularity coefficient $\beta$ of the 1/f noise model. On 104 ROI extracted from 26 FFDM mammograms, $\beta \in[1.32 ; 1.44]$, which correspond to 
$H \in[0.33 ; 0.42]$. Moreover, the line-based estimates of the minimal Hurst in both directions have equivalent empirical distributions and are approximately equal on each image. This observation is consistent with the theoretical result showing that line-based restrictions of AFBF have same Hurst indices in all directions. This gives an indirect evidence of the adequacy of the AFBF model to mammogram textures.

Moreover, we observe that horizontal and vertical Hurst index estimates have similar distributions. Standard deviations of horizontal and vertical Hurst indices are about 0.15 and their averages are 0.45 and 0.55 , respectively. On average, the mammograms seems slightly smoother in the direction parallel to the chest wall than in the perpendicular one. Besides, ranges of minimal and directional Hurst indices are not the same. This is partly due to differences in precision of index estimators. However, since the range difference is above the precision, this also indicates a texture anisotropy.

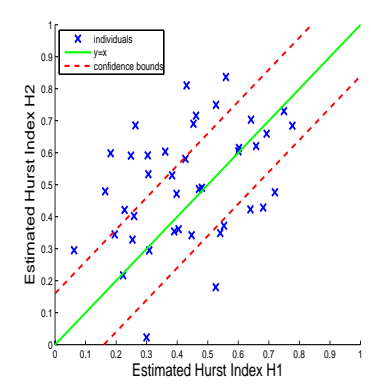

(a)

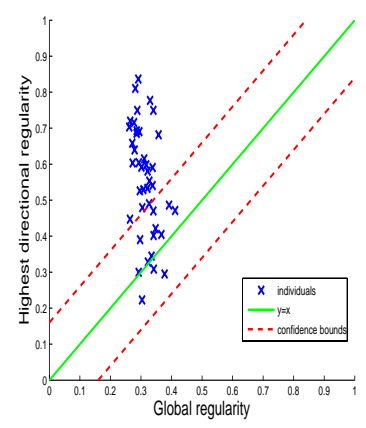

(b)

Figure 7. (a) $\left(\hat{h}_{1}^{2}, \hat{h}_{2}^{2}\right)$. (b) $\left(\min \left(\hat{H}_{01}, \hat{H}_{02}\right), \max \left(\hat{h}_{1}^{2}, \hat{h}_{2}^{2}\right)\right)$

The mammogram anisotropy is further confirmed by results shown on Figure 7 (a) and (b) and Figure 8. On these figures, we plotted histograms of estimators which are used in the different anisotropy tests, and represented the rejection bounds of these tests by red dash lines.

Similarly to $[8,12,13]$, our experiments confirm the relevance of fractional Brownian models for the characterization of the mammogram density. However, they also reveal that the isotropy assumption which is behind the mammogram modeling of $[8,12,13]$ is not valid in many cases: around 60 percents of the mammogram textures 

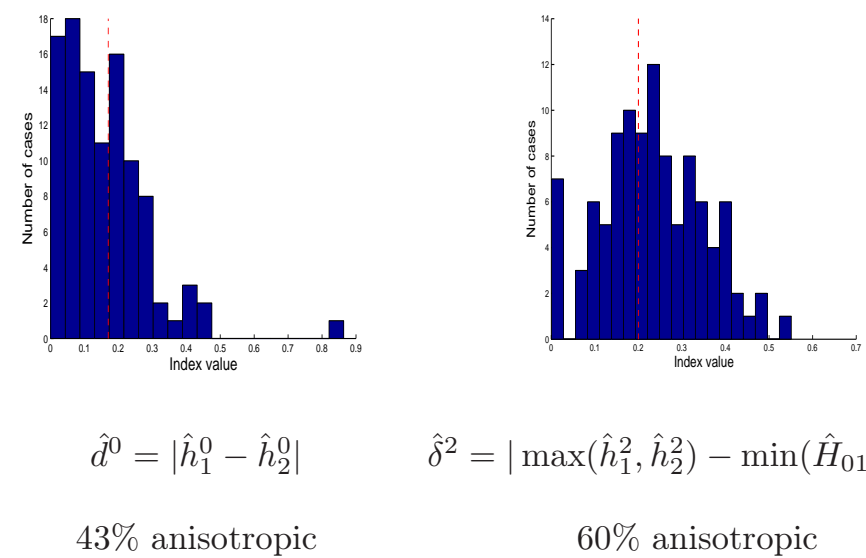

FiguRE 8. Histograms of Hurst index difference estimators

we studied could be considered as anisotropic with a high level of confidence. Hence, we conclude that AFBF model is more realistic and relevant than the isotropic FBF model for the description of FFDM textures.

\subsection{Trabecular bone x-ray images}

Results of [4] suggest that fractal analysis of radiographic trabecular bone radiographic images is a good indicator of the alteration of the bone microarchitecture. In association with bone mineral density, fractal analysis improves the fracture risk evaluation. However, since this analysis is based on an isotropic model, it does not reveal bone texture anisotropy which is of special interest for the diagnosis of osteoporosis [7,10].

In this section, we consider our estimators on trabecular bone x-ray images. Our study involved 291 cases of post menopausal women, 181 were considered as control cases and 110 as osteoporotic fracture cases. The images have been produced in the INSERM U658 (Orleans, France). The radiographs were obtained on calcaneus with a direct digital X-ray prototype $\left(\mathrm{BMA}^{\mathrm{TM}}\right.$, D3A Medical Systems, Orleans, France) having the following characteristics: focal distance $1.15 \mathrm{~m}$ and X-ray parameters $55 \mathrm{kV}$ and $20 \mathrm{mAs}$. The high-resolution digital detector integrated into the device prototype had a $50 \mu \mathrm{m}$ pixel size, providing a spatial resolution of 8 line pairs per millimeter at $10 \%$ modulation transfer function [18]. In each image, we considered a region of interest (ROI) of size $400 \times 400$ pixels. As illustrated on Figure 9, we then apply a high-pass filter to images. This filter is intended to remove the local trend of the images and increase the homogeneity of images in low frequencies. 
Indeed, we observed that low frequency inhomogeneities could cause sharp variations in Radon transforms and reduce significantly the regularity of these projections. The filtered image was obtained by substracting the image convolved with a gaussian low-pass filter to the original image.
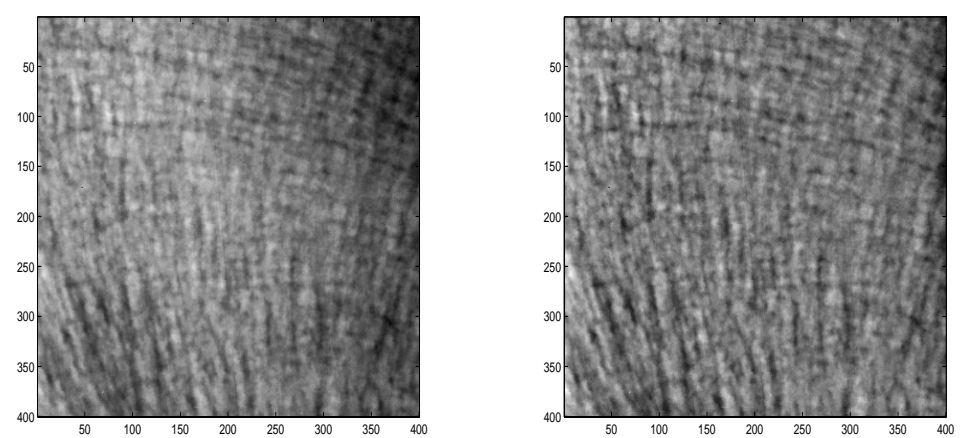

Figure 9. (a) raw image, (b) filtered image.

The estimates of the averaged Hurst index $\hat{H}_{\text {mean }}=\frac{\hat{H}_{01}+\hat{H}_{02}}{2}$ we obtained using line-based estimators on the extracted ROI are in the interval [0.484, 0.783$]$, with an average of 0.66 and a standard deviation of 0.050 . We found $\hat{H}_{\text {mean }}=0.653 \pm 0.051$ on 110 osteoporotic cases versus $\hat{H}_{\text {mean }}=0.665 \pm 0.048$ on 181 normal cases. These results are close to those obtained in previous studies, using other estimators. In [4], the authors found $H_{\text {mean }}=0.679 \pm 0.053$ on 107 fracture cases versus $H_{\text {mean }}=0.696 \pm 0.030$ on 197 control cases. Their estimation is based on the x-ray images modeling by isotropic fractional Brownian field. The Hurst parameter is estimated by an oriented fractal analysis [3]: following 36 directions, 100-300 parallel lines are modeled by fractional Brownian motion for which the Hurst parameter is estimated by the maximum likelihood estimator [19]. For each images a mean parameter $H_{\text {mean }}$ is obtained by averaging the parameters estimated in the 36 directions.

As shown in Figure 10, line-based estimates of the Hurst index in horizontal direction $\hat{H}_{01}$ is significantly greater than those in vertical direction $\hat{H}_{02}$. Since line-based restrictions of AFBF have same Hurst indices in all directions, this result shows that bone radiographs cannot be exactly considered as realizations of AFBF realizations. Such a result can be viewed as a direct evidence of the image anisotropy. 

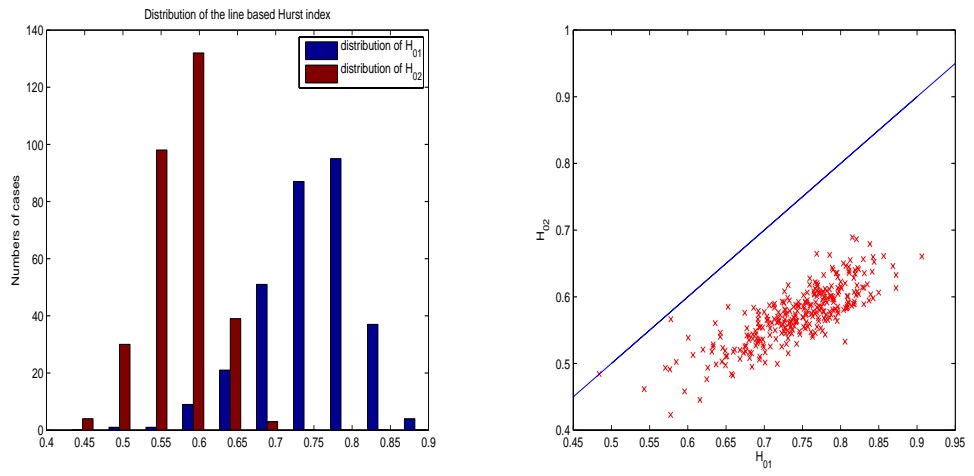

Figure 10. The Hurst indices estimated in the horizontal and vertical directions with increment step $\hat{H}_{\text {mean }}=\frac{\hat{H}_{01}+\hat{H}_{02}}{2}=0.659 \pm 0.05$.

The bones radiographs anisotropy is further confirmed by results shown on Figure 11 (a) and (b). We computed the discrete row- and column-wise projections of each ROI and the estimates of the directional Hurst indices in both directions $\left(\hat{h}_{1}^{\nu}, \hat{h}_{2}^{\nu}\right.$ given by $\left.(21)\right)$. Due to the size of the images it seems that less biased values are obtained for $\nu=1: \hat{h}_{1}^{1}=0.809 \pm 0.190$ and $\hat{h}_{2}^{1}=0.662 \pm 0.105$.

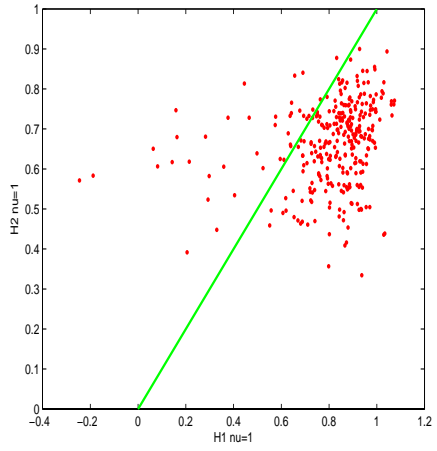

(a)

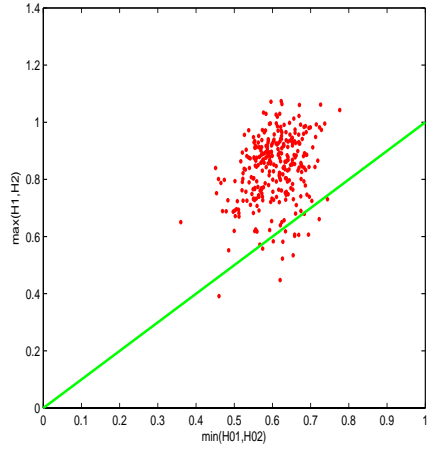

(b)

Figure 11. (a) $\left(\hat{h}_{1}^{1}, \hat{h}_{2}^{1}\right) \cdot$ (b) $\left(\min \left(\hat{H}_{01}, \hat{H}_{02}\right), \max \left(\hat{h}_{1}^{1}, \hat{h}_{2}^{1}\right)\right)$

These preliminary results emphasize the anisotropic nature of bones x-ray images. We believe that our statistical tools developed for AFBF estimation should also be helpful for bone radiographs characterization. 
This work was partially supported by ANR mipomodim ANR-05-BLAN-0017.

\section{REFERENCES}

[1] R. J. Adler. The Geometry of Random Field. John Wiley \& Sons, 1981.

[2] A. Ayache, A. Bonami, and A. Estrade. Identification and series decomposition of anisotropic Gaussian fields. Proceedings of the Catania ISAAC05 congress, 2005.

[3] C.L. Benhamou, E. Lespessailles, G. Jacquet, et al. Fractal organization of trabecular bone images on calcaneus radiographs. Journal of Bone and Mineral Research, 9:1909-1918, 1994.

[4] C.L. Benhamou, S. Poupon, E. Lespessailles, et al. Fractal analysis of radiographic trabecular bone texture and bone mineral density: two complementary parameters related to osteoporotic fractures. Journal of Bone and Mineral Research, 16(4):697$703,2001$.

[5] H. Biermé and F. Richard. Estimation of anisotropic gaussian fields through radon transform. ESAIM: Probability and Statistics, 2007.

[6] A. Bonami and A. Estrade. Anisotropic analysis of some Gaussian models. J. Fourier Anal. Appl., 9:215-236, 2003.

[7] B. Brunet-Imbault, G. Lemineur, C. Chappard, et al. A new anisotropy index on trabecular bone radiographic images using the fast Fourier transform. BMC Med. Imaging, 5(4), 2005.

[8] A. Burgess, F. Jacobson, and P. Judy. Human observer detection experiments with mammograms and power-law noise. Med. Phys., 28(4):419-437, 2001.

[9] C. Caldwell, S. Stapleton, D. Holdsworth, et al. Characterisation of mammographic parenchymal patterns by fractal dimension. Phys. Med. Biol., 35(2):235-247, 1990.

[10] C. Chappard, B. Brunet-Imbault, G. Lemineur, et al. Anisotropy changes in post-menopausal osteoporosis: characterization by a new index applied to trabecular bone radiographic images. Osteoporos Int, 16: 1193-1202, 2005.

[11] K. J. Falconer. Fractal Geometry. John Wiley \& Sons, 1990.

[12] J. Heine, S. Deine, R. Velthuizen, et al. On the statistical nature of mammograms. Med. Phys., 26(11):2254-2269, 1999.

[13] J. Heine and R. Velthuizen. Spectral analysis of full field digital mammography data. Med. Phys., 29(5):647-661, 2002.

[14] J. Istas and G. Lang. Quadratic variations and estimation of the local Holder index of a Gaussian process. Ann. Inst. Henri Poincaré, Prob. Stat., 33(4):407-436, 1997.

[15] I. Karatzas and E. Shreve. Brownian Motion and Stochastic Calculus. Springer-Verlag, 1998.

[16] J.T. Kent and A.T.A. Wood. Estimating the fractal dimension of a locally self-similar Gaussian process by using increments. J. Roy. Statist. Soc. Ser. B, 59(3):679-699, 1997. 
[17] P. Kestener, J.-M. Lina, P. Saint-Jean, et al. Wavelet-based multifractal formalism to assist in diagnosis in digitized mammograms. Image Anal. Stereol., 20:169-174, 2001.

[18] E. Lespessailles, C. Gadois, G. Lemineur, J.P. Do-Huu, L. Benhamou Bone texture analysis on direct digital radiographic images: precision study and relationship with bone mineral density at the os calcis. Calcif Tissue Int 200\%; 80: 9-102, 2007.

[19] T. Lundahl, W.J. Ohley, S.M. Kay, and R. Siffe. Fractional brownian motion: a maximum likelihood estimator and its application to image texture. IEEE Trans. on Medical Images, 5(3):152-161, 1986.

[20] B. B. Mandelbrot and J. Van Ness. Fractional Brownian motion, fractionnal noises and applications. SIAM Review, 10:422-437, 1968.

[21] A.G. Ramm and A.I. Katsevich The Radon Transform and Local Tomography. CRC Press, 1996.

[22] F. Richard and H. Biermé, A statistical methodology for testing the anisotropy of Brownian textures with an application to full-field digital mammography, http://hal.archives-ouvertes.fr/hal-00177770/fr/, preprint, 2007.

[23] M.L. Stein. Fast and exact simulation of fractional Brownian surfaces. J. Comput. Graph. Statist., 11(3):587-599, 2002. 